Journal of Universal Mathematics

Vol.2 No.1 PP.89-97 (2019)

ISSN-2618-5660

\title{
H-GROUP STRUCTURE ON INTUITIONISTIC FUZZY TOPOLOGICAL SPACE
}

\author{
SIBEL DEMIRALP AND GÜLNUR HAÇAT
}

\begin{abstract}
In this study we investigate some properties of the pointed intuitionistic fuzzy topological spaces. Then some properties of intuitionistic fuzzy loop spaces are investigated Finally it is shown that an intuitionistic fuzzy loop space is an H-group.
\end{abstract}

\section{INTRODUCTION}

In [1] Attanasov introduced the concept of intuitionistic fuzzy set as a generalization of the concept of fuzzy set, defined by Zadeh [10]. After that, some basic concepts from general topology and algebraic topology were applied to intuitionistic fuzzy sets, $[1,4]$. The concept of intuitionistic fuzzy homotopy was defined in [2] by E.A. Az-Zo'bi. In [], intuitionistic fuzzy topological group was defined.

In this study some properties of intuitionistic fuzzy topological groups. We show that the set of homotopy classes of all intuitionistic fuzzy continuous functions from a pointed IF topological space to on IF-topological group is a group. Then we prove that there exist a contravariant functor from the homotopy category of pointed IF topological spaces to the category of groups and homomorphism. Finally we establish a H-group structure on IF loop space.

\section{Preliminaries}

The notation of fuzzy set was introduced by Zadeh in [10] A fuzzy set in a nonempty set $X$ is a function $\mu_{A}: X \rightarrow[0,1]$, for any $x \in X$. For $x \in X, \mu_{A}(x)$ is the degree of membership of $x$.

Atanassov defined intuitionistic fuzzy sets in [1]. Let $X$ be a nonempty set. An intuitionistic fuzzy set (IFS) $\mathrm{A}$ in $\mathrm{X}$ is an object having the form

$$
A=\left\{\left\langle x, \mu_{A}(x), \gamma_{A}(x)\right\rangle: x \in X\right\}
$$

where the functions $\mu_{A}(x): X \rightarrow[0,1], \gamma_{A}: X \rightarrow[0,1]$ denotes the degree of membership and the degree of non-membership of each element $x \in X$ to the set $A$ respectively, and $0 \leq \mu_{A}(x)+\gamma_{A}(x) \leq 1$ for all $x \in X$.

Date: Review January 2, 2019, accepted January 22, 2019.

2000 Mathematics Subject Classification. Primary 03E72; Secondary 54A40, 55Q05,55P35.

Key words and phrases. H-group, Intuitionistic fuzzy topological space, Pointed intuitionistic fuzzy topological spaces, Intuitionistic fuzzy loop space. 
Denote the set of all intuitionistic fuzzy sets in $\mathrm{X}$ by $\operatorname{IFS}(\mathrm{X})$. Let $A$ and $B$ be IFSs of the form

$$
A=\left\{\left\langle x, \mu_{A}(x), \gamma_{A}(x)\right\rangle \mid x \in X\right\} \text { and } B=\left\{\left\langle x, \mu_{B}(x), \gamma_{B}(x)\right\rangle \mid x \in X\right\} .
$$

Then $A \subseteq B$ if and only if $\mu_{A}(x) \leq \mu_{B}(x)$ and $\gamma_{A}(x) \geq \gamma_{B}(x)$ for all $x \in X$. The intersection and the composition of $A$ and $B$ are defined as

$A \cap B=\left\{\left\langle x, \mu_{A}(x) \wedge \mu_{B}(x), \gamma_{A}(x) \vee \gamma_{B}(x)\right\rangle \mid x \in X\right\}$ and

$A \cup B=\left\{\left\langle x, \mu_{A}(x) \vee \mu_{B}(x), \gamma_{A}(x) \wedge \gamma_{B}(x)\right\rangle \mid x \in X\right\}$, respectively.

We shall use the notation $A=\left\langle\mu_{A}, \gamma_{A}\right\rangle$ instead of

$$
A=\left\{\left\langle x, \mu_{A}(x), \gamma_{A}(x)\right\rangle \mid x \in X\right\}
$$

and let $0_{\sim}=\langle 0,1\rangle$ and $1_{\sim}=\langle 1,0\rangle$.

Definition 2.1. [7] Let $r, s \in I, r+s \leq 1$ and let $A \in \operatorname{IFS}(X)$. Then $A$ is called an intuitionistic fuzzy point (IFP) with the support $p \in X$ and the value $r$ and the nonvalued $s$ if for each $y \in X$

$$
A(y)=\{.(r, s) \quad \text { if } \quad y=p,(0,1) \quad \text { if } y \neq p
$$

In this case, we write $A=p_{(r, s)}$. If $r<\mu_{B}(p)$ and $s>\gamma_{B}(p), p_{(r, s)}$ is said to be contained in $B \in \operatorname{IFS}(X)\left(p_{(r, s)} \in B\right.$ for short).

Definition 2.2. [4] An intuitionistic fuzzy topology (IFT for short) on a set $X$ is a family $\tau$ of IFSs in $X$ which satisfies the following conditions:

i) $0_{\sim}, 1_{\sim} \in \tau$

ii) If $A_{1}, A_{2} \in \tau$ then $A_{1} \cap A_{2} \in \tau$

iii) If $A j \in \tau$ for all $j \in J$ (where $J$ is an index set) then $\bigcup_{j \in J} A j \in \tau$.

Then the pair $(X, \tau)$ is called an intuitionistic fuzzy topological space (IF topological space). Every member of $\tau$ is called an intuitionistic fuzzy open set (IF open set) in $X$. The complement $A^{c}$ of an IF open set $A$ in $(X, \tau)$ is called an intuitionistic fuzzy closed set (IF closed set) in $X$.

Definition 2.3. [2] Let $(X, \tau)$ and $\left(Y, \tau^{\prime}\right)$ be two IF topological space and let $f: X \rightarrow Y$ be a function. If $f^{-1}(U) \in \tau$ for all $U \in \tau^{\prime}$, then $f$ is called intuitionistic fuzzy continuous function (IF continuous). The set of all IF continuous functions from $(X, \tau)$ to $\left(Y, \tau^{\prime}\right)$ is denoted by $\operatorname{IFC}(X, Y)$.

Definition 2.4. [8] Let $(X, \cdot)$ be a group and $A=\left\langle\mu_{A}, \gamma_{A}\right\rangle, B=\left\langle\mu_{B}, \gamma_{B}\right\rangle$ are two intuitionistic fuzzy sets in $X$. We define $A B$ and $B^{-1}$ by the respective formula,

i) $\mu_{A B}(x)=\sup _{x=x_{1} x_{2}} \min \left(\mu_{A}\left(x_{1}\right), \mu_{B}\left(x_{2}\right)\right)$ and $\gamma_{A B}(x)=\inf _{x=x_{1} x_{2}} \max \left(\gamma_{A}\left(x_{1}\right), \gamma_{B}\left(x_{2}\right)\right)$,

ii) $\mu_{B^{-1}}(x)=\mu_{B}\left(x^{-1}\right)$ and $\gamma_{B^{-1}}(x)=\gamma_{B}\left(x^{-1}\right)$.

Definition 2.5. [8] Let $(X, \cdot)$ be a group and $(X, \tau)$ be an IFTS. Let $A=\left\langle\mu_{A}, \gamma_{A}\right\rangle$, $B=\left\langle\mu_{B}, \gamma_{B}\right\rangle$ and $C=\left\langle\mu_{C}, \gamma_{C}\right\rangle$ be IFSs in $X .(X, \tau, \cdot)$ is called an intuitionistic fuzzy topological group or IF topological group for short, if and only if,

i) for all $x, y \in X$ and any Q-neighbourhood $C$ of an intuitionistic fuzzy point $(p q)_{(r, s)}$ there are Q-neighbourhoods $A$ of $p_{(r, s)}$ and $B$ of $q_{(r, s)}$ such that $A B \subseteq C$.

ii) for all $x \in X$ and any Q-neighbourhood $B$ of an $\operatorname{IFP}\left(p^{-1}\right)_{(r, s)}$, there exists a Q-neighbourhood $A$ of $p_{(r, s)}$ such that $A^{-1} \subseteq B$. 


\section{Main Results}

In this part, some properties of intuitionistic fuzzy topological groups are investigated. Then it is shown that an intuitionistic fuzzy loop space is an H-group.

Theorem 3.1. Let $(X, \tau)$ be an IF topological space, $\left(Y, \tau^{\prime}, \cdot\right)$ be an IF topological group with the identity element " $e$ " and $f, g \in \operatorname{IFC}(X, Y)$. Then the map

$$
f \bullet g: x \rightarrow f(x) \cdot g(x)
$$

from IF topological space $X$ to IF topological group $Y$ is IF continuous.

Proof. [5] Let $p_{(r, s)}$ be a IF point of $X$ and $V$ a IF open Q-neighbourhood of $(f \bullet g)\left(p_{(r, s)}\right)$. As $\left(Y, \tau^{\prime}, \cdot\right)$ is an IF topological group, there exist Q-neighbourhoods $V_{1}$ and $V_{2}$ of $f\left(p_{(r, s)}\right)=(f(p))_{(r, s)}$ and $g\left(p_{(r, s)}\right)=(g(p))_{(r, s)}$, respectively such that $V_{1} V_{2} \leq V$. Also, since the maps $f$ and $g$ are IF continuous, there exist IF open Q-neighbourhoods $U_{1}$ and $U_{2}$ of $p_{(r, s)}$ such that $f\left(U_{1}\right) \leq V_{1}$ and $g\left(U_{2}\right) \leq V_{2}$.

Let $q_{(t, k)} \in U_{1} \wedge U_{2}$. Then $q_{(t, k)} \in U_{1}$ and $q_{(t, k)} \in U_{2}$. So,

$$
f\left(q_{(t, k)}\right)=(f(q))_{(t, k)} \in f\left(U_{1}\right) \leq V_{1} \Rightarrow f\left(q_{(t, k)}\right) \in V_{1}
$$

and

Therefore,

$$
g\left(q_{(t, k)}\right)=(g(q))_{(t, k)} \in f\left(U_{2}\right) \leq V_{2} \Rightarrow g\left(q_{(t, k)}\right) \in V_{2} .
$$

Also,

$$
\begin{aligned}
& t \leq \mu_{V_{1}}(f(q)) \text { and } k \geq \gamma_{V_{1}}(f(q)) \\
& t \leq \mu_{V_{2}}(f(q)) \text { and } k \geq \gamma_{V_{2}}(f(q))
\end{aligned}
$$

$$
\begin{aligned}
& \mu_{V_{1} V 2}(f(q) g(q))=\sup \left\{\min \left\{\mu_{V_{1}}\left(f\left(q_{1}\right)\right), \mu_{V_{2}}\left(g\left(q_{2}\right)\right)\right\} \mid w_{1} w_{2}=f(q) g(q)\right\} \geq t \\
& \gamma_{V_{1} V 2}(f(q) g(q))=\inf \left\{\max \left\{\gamma_{V_{1}}\left(f\left(q_{1}\right)\right), \gamma_{V_{2}}\left(g\left(q_{2}\right)\right)\right\} \mid w_{1} w_{2}=f(q) g(q)\right\} \leq k
\end{aligned}
$$

So

$$
\begin{gathered}
r \leq \mu_{V_{1} V 2}(f(q) g(q)) \leq \mu_{V}(f(q) g(q)) \\
s \geq \gamma_{V_{1} V 2}(f(q) g(q)) \leq \gamma_{V}(f(q) g(q)) .\left(V_{1} V_{2}\right)(f(q) g(q)) \leq V(f(q) g(q))
\end{gathered}
$$

and therefore $\left((f(q) g(q))_{(t, k)}=(f \bullet g)\left(q_{(t, k)}\right) \in V\right.$. So $(f \bullet g)\left(U_{1} \wedge U_{2}\right) \leq V$. Consequently $(f \bullet g)$ is IF continuous.

Definition 3.2. [8] Let $(X, \tau)$ be an IF topological space and for $\alpha, \beta \in[0,1],(\alpha \beta)^{*}$ be an IFS defined as $(\alpha \beta)^{*}=\left\{\left\langle x, \mu_{(\alpha \beta)^{*}}, \gamma_{(\alpha \beta)^{*}}\right\rangle \mid x \in X\right\}$, where $\mu_{(\alpha \beta)^{*}}(x)=\alpha$ and $\gamma_{(\alpha \beta)^{*}}(x)=\beta$, such that $\mu_{(\alpha \beta)^{*}}(x)+\gamma_{(\alpha \beta)^{*}}(x)=1$ for every $x \in X$. If $(\alpha \beta)^{*} \in \tau$ for every $\alpha, \beta \in[0,1]$, then $(X, \tau)$ is called a fully stratified space.

Theorem 3.3. Let IF topological space $(X, \tau)$ be fully stratified, $(Y, \cdot)$ be a group with the identity element " $e$ " and $\left(Y, \tau^{\prime}, \cdot\right)$ an IF topological group. Then the map $c:(X, \tau) \rightarrow\left(Y, \tau^{\prime}, \cdot\right)$ defined such that $c(x)=e$, for every $x \in X$, is IF continuous.

Proof. Let $V \in \tau^{\prime}$. Then

$$
\begin{aligned}
(c)^{-1}(V)(x) & =<x,(c)^{-1}\left(\mu_{V}\right)(x),(c)^{-1}\left(\gamma_{V}\right)(x)> \\
= & <x, \mu_{V}(c(x)), \gamma_{V}((c)(x))> \\
= & <x, \mu_{V}(e), \gamma_{V}(e)> \\
= & V(e)
\end{aligned}
$$

for every $x \in X$. So $(c)^{-1}(V)$ is a constant IFS. Since, $(X, \tau)$ is a fully stratified IFTS, then $(c)^{-1}(V) \in \tau$. Consequently " $c$ " is IF continuous. 
Theorem 3.4. Let $(X, \tau)$ be a fully stratified IF topological space and $\left(Y, \tau^{\prime}, \cdot\right)$ be a IF topological group. Then the pair $(\operatorname{IFC}(X, Y), \bullet)$ is a group. Also if the group $(Y, \cdot)$ is abelian, then the group $(\operatorname{IFC}(X, Y), \bullet)$ is abelian.

Proof. Let $f, g, h \in \operatorname{IFC}(X, Y)$. Then

$$
\begin{aligned}
((f \bullet g) \bullet h)(x) & =(f \bullet g)(x) \cdot h(x)=(f(x) \cdot g(x)) \cdot h(x) \\
& =f(x) \cdot(g(x) \cdot h(x))=f(x) \cdot(g \bullet h)(x) \\
& =(f \bullet(g \bullet h))(x)
\end{aligned}
$$

for every $x \in X$. So $(f \bullet g) \bullet h=f \bullet(g \bullet h)$. Also $f \bullet c=c \bullet f=f$ and $f \bullet f^{-1}=f^{-1} \bullet f=c$. So $c$ is the identity element and $f^{-1} \in \operatorname{IFC}(X, Y)$ is the inverse of $f$. Consequently, $(\operatorname{IFC}(X, Y), \bullet)$ is a group. Let $(Y, \cdot)$ be an abelian group and $f, g \in \operatorname{IFC}(X, Y)$. Then,

$$
(f \bullet g)(x)=f(x) \cdot g(x)=g(x) \cdot f(x)=(g \bullet f)(x)
$$

for all $x \in X$.

Let $\left(X, p_{(r, s)}\right)$ be a pointed IF topological space and $\left(Y, \cdot, e_{(r, s)}\right)$ be an IF topological group. Then the set of homotopy classes of all IF contmuous functions from $\left(X, p_{(r, s)}\right)$ to $\left(Y, \cdot, e_{(r, s)}\right)$ is denoted by $\left[\left(X, p_{(r, s)}\right) ;\left(Y, \cdot, e_{(r, s)}\right)\right]$.

Theorem 3.5. $\left[\left(X, p_{(r, s)}\right) ;\left(Y, \cdot, e_{(r, s)}\right)\right]$ is a group under the product $[f] \otimes[g]=$ $[f \bullet g]$. Also if $(Y, \cdot)$ is abelian then $\left(\left[\left(X, p_{(r, s)}\right) ;\left(Y, \cdot, e_{(r, s)}\right)\right], \otimes\right)$ is abelian.

Proof. i) Let $[f],[g],[h] \in\left[\left(X, p_{(r, s)}\right) ;\left(Y, \cdot, e_{(r, s)}\right)\right]$, then

$$
\begin{aligned}
(([f] \otimes[g]) \otimes[h]) & =([f \bullet g] \otimes[h])=([(f \bullet g) \bullet h]) \\
& =([f \bullet(g \bullet h)])=([f] \otimes[g \bullet h]) \\
& =([f] \otimes([g] \otimes[h])) .
\end{aligned}
$$

So $\otimes$ is associative.

ii) For all $[f] \in\left[\left(X, p_{(r, s)}\right) ;\left(Y, \cdot, e_{(r, s)}\right)\right],[f] \otimes[c]=[f \bullet c]=[f]$ and $[c] \otimes[f]=$ $[c \bullet f]=[f]$. So $[c]$ is the identity element.

iii)For all $[f] \in\left[\left(X, p_{(r, s)}\right) ;\left(Y, \cdot, e_{(r, s)}\right)\right],[f] \otimes\left[f^{-1}\right]=\left[f \bullet f^{-1}\right]=[c]$ and $\left[f^{-1}\right] \otimes$ $[f]=\left[f^{-1} \bullet f\right]=[c]$. So $\left[f^{-1}\right]$ is the inverse of $[f]$, i.e. $\left[f^{-1}\right]=[f]^{-1}$.

Let $(Y, \cdot)$ be abelian, then the group $(\operatorname{IFC}(X, Y), \bullet)$ is abelian. So for $[f],[g] \in$ $\left[\left(X, p_{(r, s)}\right) ;\left(Y, \cdot, e_{(r, s)}\right)\right]$,

$$
[f] \otimes[g]=[f \bullet g]=[g \bullet f]=[g] \otimes[f] .
$$

The category whose objects are pointed IF topological spaces and morphisms are

$$
\operatorname{hom}\left(X, p_{(r, s)}\right),\left(X^{\prime}, p_{(r, s)}^{\prime}\right)=\left[\left(X, p_{(r, s)}\right),\left(X^{\prime}, p_{(r, s)}^{\prime}\right)\right]
$$

is called the homotopy category of pointed IF topological spaces.

Theorem 3.6. [6] For any category $C$ and object $Y$ of $C$, there is a contravariant functor $\Pi^{Y}$ from the category of $C$ to the category of sets and functions which associates to an object $X$ of $C$ the set $\Pi^{Y}(X)=\operatorname{hom}(X, Y)=\{f \mid f: X \rightarrow Y\}$ and to a morphism $f: X \rightarrow X^{\prime}$ the function $\Pi^{Y}(f)=f^{*}: \operatorname{hom}\left(X^{\prime}, Y\right) \rightarrow(X, Y)$ defined by $f^{*}\left(g^{\prime}\right)=g^{\prime} \circ f$, for $g^{\prime}: X^{\prime} \rightarrow Y$. 
Theorem 3.7. There exist a contravariant functor from the homotopy category of pointed IF topological spaces to the category of groups and homomorphisms.

Proof. Let $\left(Y, \cdot, e_{(r, s)}\right)$ be an IF topological group. We show that if $\left(X, p_{(r, s)}\right)$ and $\left(X^{\prime}, p_{(r, s)}^{\prime}\right)$ are two objects and $[f] \in\left[\left(X, p_{(r, s)}\right),\left(X^{\prime}, p_{(r, s)}^{\prime}\right)\right]$ is a morphism of the homotopy category of pointed IF topological spaces, then $\Pi^{Y}\left(X, p_{(r, s)}\right)$ is a group and $\Pi^{Y}([f])$ is an homomorphism. $\Pi^{Y}\left(X, p_{(r, s)}\right)=\operatorname{hom}\left(\left(X, p_{(r, s)}\right),\left(Y, \cdot, e_{(r, s)}\right)\right)=$ $\left[\left(X, p_{(r, s)}\right),\left(Y, \cdot, e_{(r, s)}\right)\right]$. So $\Pi^{Y}\left(X, p_{(r, s)}\right)$ is a group. $\Pi^{Y}([f])=f^{*}: \operatorname{hom}\left(\left(X^{\prime}, p_{(r, s)}^{\prime}\right),\left(Y, \cdot, e_{(r, s)}\right)\right) \rightarrow$ $\operatorname{hom}\left(\left(X, p_{(r, s)}\right),\left(Y, \cdot, e_{(r, s)}\right)\right) \Rightarrow f^{*}:\left[\left(X^{\prime}, p_{(r, s)}^{\prime}\right),\left(Y, \cdot, e_{(r, s)}\right)\right] \rightarrow\left[\left(X, p_{(r, s)}\right),\left(Y, \cdot, e_{(r, s)}\right)\right]$

is a function such that, for any $[g] \in\left[\left(X^{\prime}, p_{(r, s)}^{\prime}\right),\left(Y, \bullet, q_{(r, s)}\right)\right], f^{*}([g])=[g] \circ f=$ $[g \circ f]$.

As $\left[\left(X^{\prime}, p_{(r, s)}^{\prime}\right),\left(Y, \cdot, e_{(r, s)}\right)\right]$ and $\left[\left(X, p_{(r, s)}\right),\left(Y, \cdot, e_{(r, s)}\right)\right]$ are groups, $f^{*}$ is a morphism between groups.

Let $\left[g^{\prime}\right],\left[g^{\prime \prime}\right] \in\left[\left(X^{\prime}, p_{(r, s)}^{\prime}\right),\left(Y, \cdot, e_{(r, s)}\right)\right]$. Then,

$$
\begin{aligned}
f^{*}\left(\left[g^{\prime}\right] \otimes\left[g^{\prime \prime}\right]\right) & =f^{*}\left(\left[g^{\prime} \bullet g^{\prime \prime}\right]\right)=\left[\left(g^{\prime} \bullet g^{\prime \prime}\right) \circ f\right] \\
& =\left[\left(g^{\prime} \circ f\right) \bullet\left(g^{\prime \prime} \circ f\right)\right]=\left[g^{\prime} \circ f\right] \otimes\left[g^{\prime \prime} \circ f\right] \\
& =f^{*}\left(\left[g^{\prime}\right]\right) \otimes f^{*}\left(\left[g^{\prime \prime}\right]\right) .
\end{aligned}
$$

So $f^{*}$ is homomorphism.

Let $\left[1_{X}\right]$ be the unit morphism of $\left[\left(X, p_{(r, s)}\right),\left(X^{\prime}, p_{(r, s)}^{\prime}\right)\right]$. Then $\Pi^{Y}\left(\left[1_{X}\right]\right)([h])=$ $1_{X}^{*}([h])=\left[h \circ 1_{X}\right]=[h]$. So $\Pi^{Y}\left(\left[1_{X}\right]\right)$ is the unit element.

Let $\varphi \in\left[\left(X^{\prime}, p_{(r, s)}^{\prime}\right),\left(X^{\prime \prime}, p_{(r, s)}^{\prime \prime}\right)\right]$. For any morphism $h^{\prime} \in\left[\left(X^{\prime \prime}, p_{(r, s)}^{\prime \prime}\right),\left(Y, \cdot, e_{(r, s)}\right)\right]$,

$$
\begin{aligned}
\Pi^{Y}([\varphi])\left(\left[h^{\prime}\right]\right) & =\left[h^{\prime} \circ \varphi\right] \\
\Pi^{Y}([f])\left(\left[h^{\prime} \circ \varphi\right]\right) & =\left[\left(h^{\prime} \circ \varphi\right) \circ f\right] \\
& =\left[h^{\prime} \circ(\varphi \circ f)\right] \\
& =\Pi^{Y}([\varphi \circ f])\left(\left[h^{\prime}\right]\right) .
\end{aligned}
$$

Thus $\Pi^{Y}([f])\left(\left[h^{\prime} \circ \varphi\right]\right)=\Pi^{Y}([f])\left(\Pi^{Y}([\varphi])\left(\left[h^{\prime}\right]\right)\right)=\left(\Pi^{Y}([f]) \circ \Pi^{Y}([\varphi])\right)\left(\left[h^{\prime}\right]\right)$. So $\Pi^{Y}([f])=\Pi^{Y}([f]) \circ \Pi^{Y}([\varphi])$. Consequently $\Pi^{Y}$ is a contravariant functor.

Definition 3.8. [2] Let $(X, T)$ be a topological space.Then

$$
\tilde{\tau}=\{A \in \operatorname{IFP}(X) \mid \operatorname{Supp} A \in T\}
$$

is an intuitionistic fuzzy topology on $X$, called the intuitionistic fuzzy topology on $X$ introduced by $T$ and $(X, \tilde{\tau})$ is called the intuitionistic fuzzy topological space introduced by $(X, T)$.

Let $\varepsilon_{I}$ denote Euclidean subspace topology on $I$ and $\left(I, \widetilde{\varepsilon}_{I}\right)$ denote the IF topological space introduced by the topological space $\left(I, \varepsilon_{I}\right)$.

Definition 3.9. [2] Let $(X, \tau),\left(Y, \tau^{\prime}\right)$ be IF topological spaces and $f, g \in I F C(X, Y)$. If there exist an IF continuous function

$$
F:(X, \tau) \times\left(I, \tilde{\varepsilon}_{I}\right) \rightarrow\left(Y, \tau^{\prime}\right)
$$


such that $F(x, 0)=f(x)$ and $F(x, 1)=g(x)$, for all $x \in X$, then $f$ and $g$ are intuitionistic fuzzy homotopic. The mapping $F$ is called intuitionistic fuzzy homotopy from $f$ to $g$ and we write $f \sim g$.

The intuitionistic fuzzy homotopy relation " $\sim$ is an equivalence relation. So the set $\operatorname{IFC}(X, Y)$ is partitioned into equivalence classes under the relation " $\sim$ ". The equivalence classes are called intuitionistic fuzzy homotopy classes and the set of all IF homotopy classes of the IF continuous functions from $(X, \tau)$ to $\left(Y, \tau^{\prime}\right)$ is denoted by $[X, Y]$. The IF homotopy class of a function $f$ is denoted by $[f]$.

Definition 3.10. Let $(X, \tau)$ be an IF topological space. If there exists an IF open and IF closed set $G$ such that $G \neq 1_{\sim}$ and $G \neq 0_{\sim}$, then $X$ is said to be fuzzy $C_{5}$-disconnected. $\mathrm{X}$ is said to be fuzzy $C_{5}$ - connected if it is not fuzzy $C_{5}-$ disconnected.

Definition 3.11. [2] Let $(X, \tau)$ be an IFTS, $\alpha:\left(I, \tilde{\varepsilon}_{I}\right) \rightarrow(X, \tau)$ be a IF continuous function and $E$ is a fuzzy $C_{5}$ - connected in $\left(I, \widetilde{\varepsilon}_{I}\right)$ with $\mu_{E}(0), \mu_{E}(1)>0$ and $v_{E}(0), v_{E}(1)<1$. Then the IFS $\alpha(E)$ is called an intuitionistic fuzzy path in $(X, \tau)$. The IF points $p_{1}(\lambda, \delta)$ and $p_{2}(\gamma, \theta)$ in $(X, \tau)$ are called the initial point and the terminal point of the intuitionistic fuzzy path $\alpha(E)$ respectively.

If the initial point and the terminal point are equal, then we call the intuitionistic fuzzy path an intuitionistic fuzzy loop. We denote the collection of all intuitionistic fuzzy loops in $(X, \tau)$ by $\Omega((X, \tau), p(\lambda, \delta))$.

Definition 3.12. Let $(X, \tau)$ be a fuzzy topological space and $p(t, s)$ be a IF point in $X$. The pair $(X, p(t, s))$ is called a pointed intuitionistic fuzzy topological space and $p(t, s)$ is called the base point of $(X, p(t, s))$.

If $\left(X, p_{1}(\lambda, \delta)\right)$ and $\left(Y, p_{2}(\gamma, \theta)\right)$ are pointed IF topological spaces, then all IF continuous functions $f:\left(X, p_{1}(\lambda, \delta)\right) \rightarrow\left(Y, p_{2}(\gamma, \theta)\right)$ preserve the base point, i.e. $f\left(p_{1}(\lambda, \delta)\right)=p_{2}(\gamma, \theta)$ and that all fuzzy homotopies be relative to the base point. The homotopy classes of base point preserving functions

$$
f:\left(X, p_{1}(\lambda, \delta)\right) \rightarrow\left(Y, p_{2}(\gamma, \theta)\right)
$$

is denoted by $\left[\left(X, p_{1}(\lambda, \delta)\right) ;\left(Y, p_{2}(\gamma, \theta)\right)\right]$.

The set of all IF loops in $(X, p(r, s))$ based at $p(r, s)$ is a pointed intuitionistic fuzzy topological space with the $\omega_{p}(A)$ which equal to $p(r, s)$ at any point. We denote that space by $\Omega\left(X, \omega_{p}(A)\right)$.

Definition 3.13. [6] An H-space consists of a pointed topological space $X$ together with a continuous multiplication $m: X \times X \longrightarrow X$ for which the constant map $c: X \rightarrow X$ is a homotopy identity, i.e. $\quad m \circ\left(1_{x}, c\right) \simeq 1_{x}$ and $m \circ\left(c, 1_{x}\right) \simeq 1_{x}$ - An H-group is an $\mathrm{H}$-space whose multiplication is homotopy associative, i.e. $m \circ\left(m \times 1_{X}\right) \simeq m \circ\left(1_{X} \times m\right)$ and has a homotopy inverse, i.e. $m \circ\left(\varphi, 1_{X}\right) \simeq c \simeq$ $m \circ\left(\varphi, 1_{X}\right)$, for a map $\varphi: X \rightarrow X$.

Proposition 1. [2] Let $\alpha(E)$ ve $\beta(D)$ be IF loops in $\Omega\left(X, \omega_{p}(A)\right)$ at $p(r, s)$, then we define

$$
(E+D)(t)=\left\{\begin{array}{cc}
\mu_{E}(2 t), \gamma_{E}(1-2 t) & , 0 \leq t \leq \frac{1}{2} \\
\mu_{D}(2 t-1), \gamma_{E}(2-2 t) & , \frac{1}{2} \leq t \leq 1
\end{array}\right.
$$


is fuzzy $C_{5}-$ connected with

$$
\begin{aligned}
\mu_{(E+D)}(0), \mu_{(E+D)}(1) & >0 \\
\gamma_{(E+D)}(0), \gamma_{(E+D)}(1) & <1 .
\end{aligned}
$$

Let $h:\left(I, \widetilde{\varepsilon}_{I}\right) \rightarrow(X, \tau)$ be a IF continuous function defined by

$$
h(t)=\left\{\begin{array}{cc}
\alpha(2 t) & , 0 \leq t \leq \frac{1}{2} \\
\beta(2 t-1) & , \frac{1}{2} \leq t \leq 1
\end{array} .\right.
$$

Then

$$
\begin{aligned}
h(E+D)(x) & =\left\{\begin{array}{cl}
\alpha\left((2 t)_{E(2 t)}\right) & , 0 \leq t \leq \frac{1}{2} \\
\beta\left((2 t-1)_{D(2 t-1)}\right) & , \frac{1}{2} \leq t \leq 1
\end{array}\right. \\
& =\left\{\begin{array}{cc}
\bigvee_{(2 t-1) \in \beta^{-1}(x)} \mu_{E}(2 t), \bigwedge_{D}(2 t-1), \bigwedge_{2 t \in \alpha^{-1}(x)} \gamma_{E}(2 t) & , 0 \leq t \leq \frac{1}{2} \\
\bigvee_{(2 t-1) \in \beta^{-1}(x)} \gamma_{D}(2 t-1) & , \frac{1}{2} \leq t \leq 1
\end{array} .\right.
\end{aligned}
$$

Theorem 3.14. $\Omega\left(X, \omega_{p}(A)\right)$ is an H-group.

Proof. If we define a map $m: \Omega\left(X, \omega_{p}(A)\right) \times \Omega\left(X, \omega_{p}(A)\right) \rightarrow \Omega\left(X, \omega_{p}(A)\right)$ such that $m(\alpha(E), \beta(D))=h(E+D)$ then $m$ can be thought as IF continuous multiplication of $\Omega\left(X, \omega_{p}(A)\right)$.

Let $c: \Omega\left(X, \omega_{p}(A)\right) \rightarrow \Omega\left(X, \omega_{p}(A)\right)$ be a constant map defined such that $c(\alpha(E))=\omega_{p}(A)$. Then

$$
\begin{aligned}
& \left(m \circ\left(1_{\Omega X}, c\right)(\alpha(E))(x)=m\left(\alpha(E), \omega_{p}(A)\right)(x)\right. \\
& \quad=\left\{\begin{array}{cl}
\bigvee_{2 t \in \alpha^{-1}(x)} \mu_{E}(2 t), \bigwedge_{2 t \in \alpha^{-1}(x)} \gamma_{E}(2 t) & , 0 \leq t \leq \frac{1}{2} \\
\bigvee_{(2 t-1) \in \omega_{p}^{-1}(x)} \mu_{A}(2 t-1), \bigwedge_{(2 t-1) \in \omega_{p}^{-1}(x)} \gamma_{A}(2 t-1) & , \frac{1}{2} \leq t \leq 1
\end{array}\right. \\
& =\left\{\begin{array}{cl}
\bigvee_{2 t \in \alpha^{-1}(x)} \mu_{E}(2 t), \bigwedge_{2 t \in \alpha^{-1}(x)} \gamma_{E}(2 t) & , 0 \leq t \leq \frac{1}{2} \\
(r, s) & , \frac{1}{2} \leq t \leq 1
\end{array}\right.
\end{aligned}
$$

and $\left(1_{\Omega X}\right)(\alpha(E))(t)=\alpha(E)(t)=\alpha\left(t_{E(t)}\right)$. Let $F$ be a function such that,

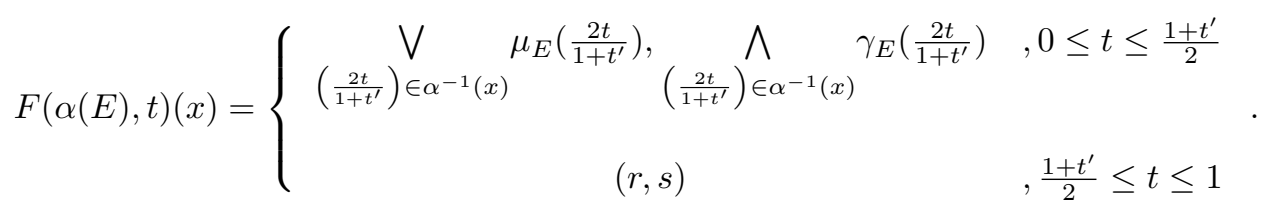

Then for all $x \in X, F(\alpha(E), 0)(x)=m(E+A)(x)=m\left(\alpha(E), \omega_{p}(A)\right)(x) . F$ is IF continuous and $m \circ\left(1_{\Omega X}, c\right) \stackrel{F}{\sim} 1_{\Omega X}$. By the same way $1_{\Omega X} \stackrel{F}{\sim} m \circ\left(c, 1_{\Omega X}\right)$. Consequently $\Omega\left(X, \omega_{p}(A)\right)$ is an Hopf space. Let show that $m$ is IF homotopy associative. 


$$
\begin{aligned}
& \left(m \circ\left(m \times 1_{\Omega X}\right)\right)(\alpha(E), \beta(D), \delta(B))(x)=m(m(\alpha(E), \beta(D)), \delta(B))(x) \\
& =\left\{\begin{array}{cl}
m(\alpha(E), \beta(D))\left((2 t)_{(E+D)(2 t)}\right) & , 0 \leq t \leq \frac{1}{2} \\
\delta\left((2 t-1)_{B(2 t-1)}\right) & , \frac{1}{2} \leq t \leq 1
\end{array}\right.
\end{aligned}
$$

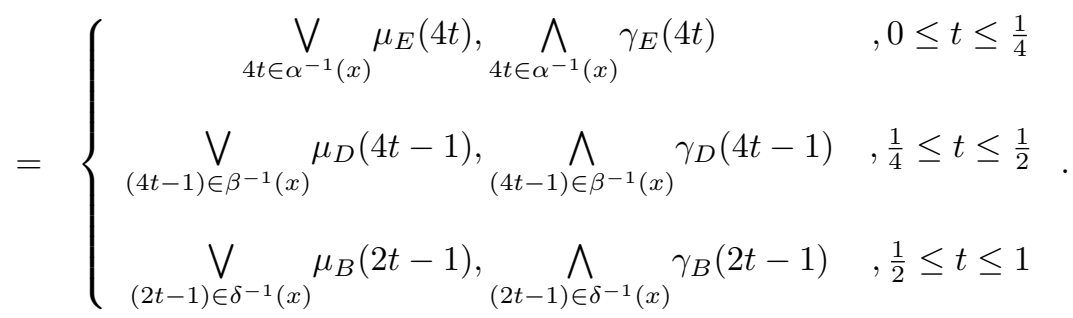

and $\left(m \circ\left(1_{\Omega X} \times m\right)\right)(\alpha(E), \beta(D), \delta(B))(x)=m(\alpha(E), m(\beta(D), \delta(B)))(x)$

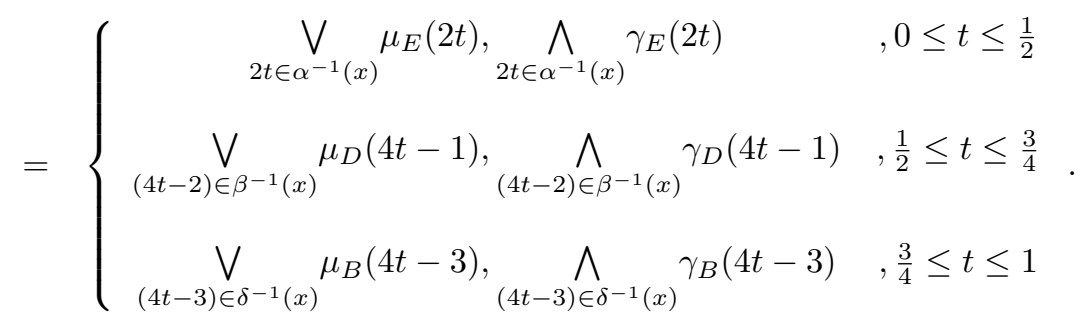

Then $\left(m \circ\left(m \times 1_{\Omega X}\right)\right) \stackrel{G}{\sim}\left(m \circ\left(1_{\Omega X} \times m\right)\right)$ by a IF homotopy

$$
G: \Omega\left(X, \omega_{p}(A)\right) \times \Omega\left(X, \omega_{p}(A)\right) \times \Omega\left(X, \omega_{p}(A)\right) \times\left(I, \widetilde{\varepsilon}_{I}\right) \rightarrow \Omega\left(X, \omega_{p}(A)\right)
$$

defined by

$$
\begin{aligned}
& G(\alpha(E), \beta(D), \delta(B), t)(x)
\end{aligned}
$$

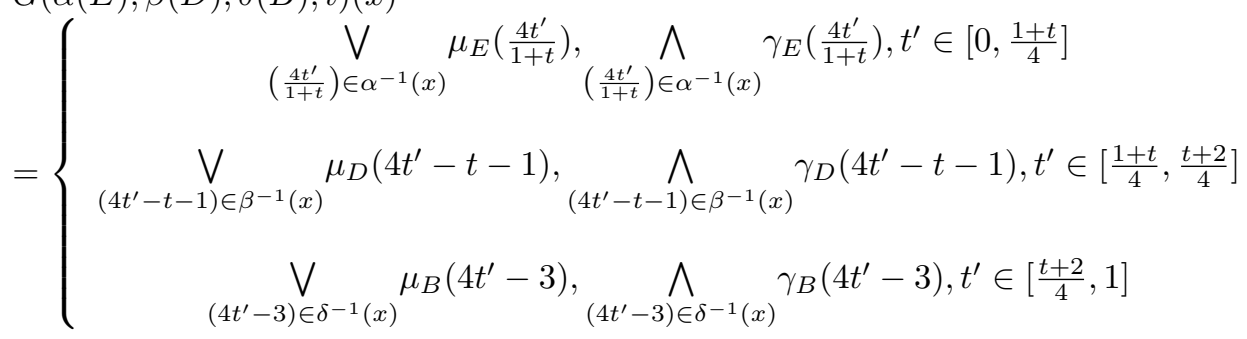

Let $\varphi: \Omega\left(X, \omega_{p}(A)\right) \rightarrow \Omega\left(X, \omega_{p}(A)\right)$ be a IF continuous function defined as $\varphi(\alpha(E))=\alpha(1-E)$ such that $(1-E)(t)=E(1-t)$. Then $\alpha\left(0_{(1-E)(0)}\right)=\alpha\left(1_{(1-E)(1)}\right)=p(t, s)$.

$\left(m \circ\left(\varphi, 1_{\Omega X}\right)\right)(\alpha(E))(t)=m(\alpha(E)(1-t), \alpha(E)(t))=m\left(\alpha^{-1}(E)(t), \alpha(E)(t)\right)$

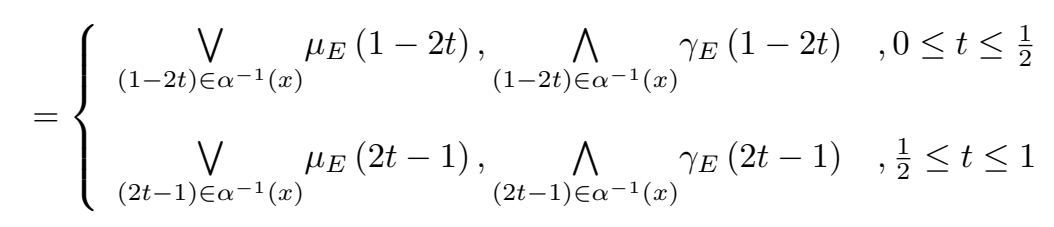


and $\left(m \circ\left(1_{\Omega X}, \varphi\right)\right)(\alpha(E))(t)=m(\alpha(E)(t), \alpha(E)(1-t))=m\left(\alpha(E)(t), \alpha^{-1}(E)(t)\right)$

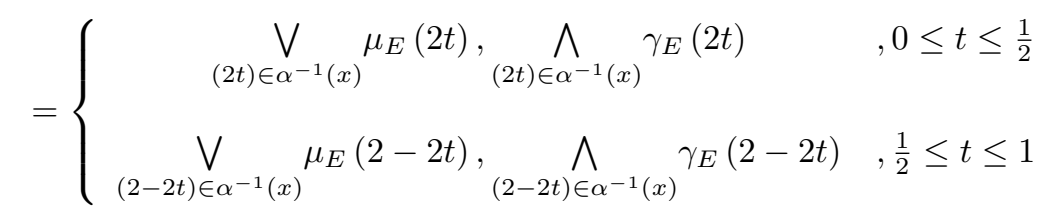

Then $\left(m \circ\left(\varphi, 1_{\Omega X}\right)\right) \stackrel{G^{\prime}}{\sim} c$ and $\left(m \circ\left(1_{\Omega X}, \varphi\right)\right) \stackrel{G^{\prime}}{\sim} c$ by a IF homotopy

$$
G^{\prime}: \Omega\left(X, \omega_{p}(A)\right) \times\left(I, \tilde{\varepsilon}_{I}\right) \rightarrow \Omega\left(X, \omega_{p}(A)\right)
$$

defined by,

$$
\begin{aligned}
& G^{\prime}(\alpha(E), t)\left(t^{\prime}\right)=
\end{aligned}
$$

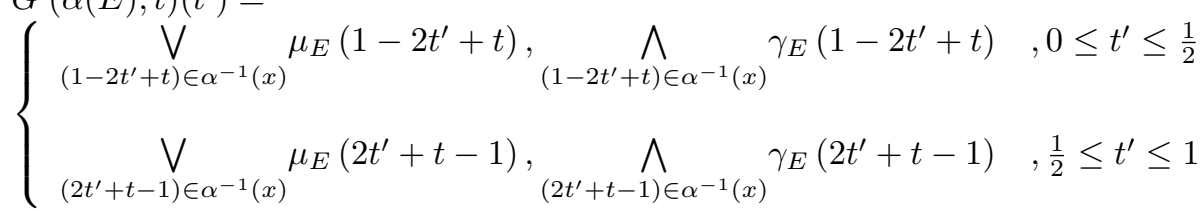

Consequently IF loop space $\Omega\left(X, \omega_{p}(A)\right)$ is a H-group.

\section{REFERENCES}

[1] Atanassov, Krassimir T. (1999). Intuitionistic fuzzy sets, Intuitionistic Fuzzy Sets. Physica, Heidelberg, 1-137.

[2] Az-Zo'bi, E. A., Marashdeh, M.F. \& Uzbashy, R.F. (2014) The Fundamental Group of Intuitionistic Fuzzy Topological Spaces, Applied Mathematical Sciences, 8.157, 7829-7843.

[3] Citil, M., \& Cuvalcıoglu, G. (2014). On intuitionistic fuzzy homotopy theory, NIFS, 2, 31-36.

[4] Çoker, D. (1997). An introduction to intuitionistic fuzzy topological spaces, Fuzzy sets and systems, 88(1), 81-89.

[5] Ganster, M., Georgiou, D. N., \& Jafari, S. (2005). On fuzzy topological groups and fuzzy continuous functions, Hacettepe Journal of Mathematics and Statistics, 34, 35-43.

[6] Hatcher, A. (2001). H-spaces and Hopf Algebras, Algebraic Topology, Cambridge University Press, Cambridge, New York.

[7] Hur, Kul, Jun-Hui Kim, \& Jang-Hyun Ryou (2004) Intuitionistic fuzzy topological spaces, The Pure and Applied Mathematics, 11.3, 243-265.

[8] Padmapriya, S., Uma, M. K., \& Roja, E. (2014). A study on intuitionistic fuzzy topological groups, Ann. Fuzzy Math. Inform, 76, 991-1004.

[9] Santhi, R., \& Jayanthi, D. (2010). Intuitionistic fuzzy generalized semi-pre closed mappings, NIFS, 16(3), 28-39.

[10] Zadeh, Lotfi A. (1996). Fuzzy sets, Fuzzy Sets, Fuzzy Logic, And Fuzzy Systems: Selected Papers by Lotfi A Zadeh, 394-432.

(1) Department of Mathematics, University of Kastamonu, [0Pt] Kastamonu, Turkey E-mail address: sdemiralp@kastamonu.edu.tr

(2) Department of Mathematics, University of Kastamonu, [0pt] Kastamonu, Turkey E-mail address: ghacat@ogr.kastamonu.edu.tr 